LBL- -25818

DE89 006604

\title{
A STUDY OF THE EFFECTS OF COPPER TO SUPERCONDUCTOR RATIO ON STABILITY*
}

\author{
W. V. Hassenzahl \\ Accelerator and Fusion Research Division \\ Lawrence Berkeley Laboratory \\ 1 Cyclotron Road \\ Berkeley, Califormia 94720
}

Presented at the "Japan-U.S. Seminar on Basic Mechanisms of Helium Heat Transfer and Related Influence on Stablity of Superconducting Magnets," August 29 - September 2, 1988, Fukuoka, Japan

*This work was supponed by the Director. Otfice of Energy Research, Office of High Energy and Nuclear Physics, High Energy Physics Division, U.S. Dept. of Energy, under Contract No. DE-AC03-76SF00098. 
W. Y. Hassenzahl

Lawrence Berkeley Laboratory

University of Califormia

Berkeley, CA 94720

\section{ABSTRACT}

Accelerator dipole magnets operate with very low copper to superconductor ratios (R). This ability to operate with low $R$ is significant in its impact on cost. The optimum value of $R$ has been an issue of considerable debate. This study shows that the minimum propagating zone length is greatest for copper to superconductor ratios well below 1.5 , which appears to be in contradiction with some of the dara

\section{INTRODUCTION}

The purpose of this presentation is to address one small area of the large question of stability of superconductors. The effect of interest here is that of the copper to superconductor matio $(R)$ on the stability of a magnet against small disturbances. The calculations will be limited to conductors operating at $4.2 \mathrm{~K}$ and having low, between 0.5 and 2.5 , copper to superconductor ratios. Clearly, unless very small in diameter and well bathed in helium, these conductors will not be cryostable. Thus, a disturbance that produces even a very small normal zone is likely to initiaie a quench.

There is considerable data on the quench characteristics of cables made of strands of various copper to supenconductor ratios from W. Sampson, et al., of BNL (1), as shown in Fig. 1. This data suggests that the larger the copper to superconductor ratio. the more stable the conductor. Though this data is presented in a rather simple way and the real picture is somewhat more complex, a major issue is how to reconcile this data with the rather intuitive belief that decreasing the ratio, i.e., adding more superconductor to increase $\mathrm{L}_{c}$, will make a conductor more stable against a quench.

- This work was supporred by the Director, Office of Energy Research, Office of High Energy and Nuclear Physics. High Energy Physics Division, U.S. Dept. of Energy, under Contract . Vo. DEA.C03-76SF00098. 
One suggestion on seeing this data might be that the results are an artifact of the measuring process and that they do not represent the performance of conductors in a real magnet. This possibility is very real because the mechanical constraints and magneric forces in the rest rig described in Ref. 1 are different from those in a real magnet. Several different configurations of conductor and levels of applied load have been used for the tests in the apparanes and there is some effect due to these variables. But this variation does not provide a satisfactory explanation for the data of Fig. 1. Sampson has suggested (and I agree) that the data supports the following conclusion (in my words), "Increasing the copper to superconductor ratio appears to contribute to the conductor being more accommodating in temis of resisting either mechanical disturbances or their effects."

The study of the quantitarive effects of the copper to superconductor ratio on conductor and coil performance presented here began some time aga. Since the first draft of this report the author has become aware of similar calculations by Tigner and Quigg(2) and No(3). This paper begins with a qualitative discussion of the effects of the copper to superconductor ratio on magnet design and performance, followed by a quantitative analysis of two relevant factors; the minimum propagating zone (MPZ) and the minimum quench energy (MQE). Usuaily when we think of margin it is with respect to the temperature or the current. For the accelerator dipole magnets it seems more appropriate to think first of the size of the (MPZ) and then of a closely related quantiry the enthaipy margin, i.e., the minimum energy required to initiate a quench. MQE. The discussion in the next section sets the stage for the calculations that follow. Its purpose is to comment on the many effects that cannot be easily included in a quantitative analysis.

QUALITATTVE DISCUSSION OF EFFECTS OF COPPER TO SUPERCONDUCTOR RATIO ON MAGNET PERFORMANCE

Before describing this analysis procedure, it is necessary to exclude some items. For the present we ignore those effects, such as heat reatments and improving homogeneity that can cause the superconductor to have a better current density. Thus, conductors are primarily considered on the same basis in terms of superconductor characteristics, though the effect of variation of critical current 
at $4.2 \mathrm{~K}$ and $5 \mathrm{~T}$ in the range $2250-3250 \mathrm{~A} / \mathrm{mm}^{2}$ will be included in the evaluation as a separate issue. The impact of homogeneity on mechanical properties, for example, is beyond the scope of this work.

The resistivity ratio of copper is affected by magnetic field. However, the variation is relatively small in the field range berween 5 and $7 \mathrm{~T}$ considered here. It does not significandy affect the relative values of MPZ or MQE. A more detailed evaluation may include explicitly the magnetoresistance, which is important for magnet design.

Let us use the conductor strand in the SSC inner layer cable as a benchmark The SSC dipole magnet reaches a normal peak field of $7 \mathrm{~T}$ at a strand current of 283 amperes. Because the MPZ is quite small, it is sufficient to consider individual strands in the 23 strand superconducting cable.

What are the possible effects of the copper to superconductor ratio on MPZ? At the high end. i.e.. no superconductor, there can be no superconducting current at all. Thus the MPZ is zero. At the other extreme, no copper at all, the SSC strand would be unstable and would cerainly be flux jump limited in the range of 2 or so Tesia Also, the minimum propagating zone would be that of a pure superconductor, some tens of microns, which for all intents and purposes is also zero. (See Ref. 5. page 76). Knowing that some copper must be present, we can search for the maximum MPZ. Various pachs are open wo this search. One is to evaluate the mechanical incegrity or a conductor with more or less copper. In fact this is very difficult to do, but it is possible to ask about the effect of $R$ on the characteristics of the conductors ar the cable edge where the strands may be considerably deformed.

Copper can be added to the conductor in 3 rather different locations: at the center of the conductor. distributed within the filaments, or on the outer surface. Copper on the outside of the filaments has some beneficial mechanical effects. First, by placing the copper here it puts the superconductor closer to the neurral axis, and thus somewhat isolated from external mechanical forces and time varying fields. Second copper here can accommodate the plastic strain at the edge of the cable 
where the conductor changes directions. Third, this copper can be deformed fairly easily in the interior of the cable where conductors cross and thus are "knuckled" while passing through the rollers of a nurks head. These knuckles hold thoughout the magnet construction. As accelerator dipoles have pushed this technology to fairly fine, 4 to 8 micron, filaments, the spacing between them has become critical. If it is too small there can be a coupling of the supercurrents between filaments, the so-called proximity effect. If it is too large then the hydrodynamic forces on the filaments during the wire drawing process causes an instability in the diameter of the filament and results in sausaging. This variation in filament diameter along the length reduces the overall current carrying capability of the conductor. As a result the addijon of copper between filaments is not a parameter that can be varied without the possibility of significant impact on conductor performance. Discussions of these effects is avoided by assuming that the variation of copper will be on the outside and/or center of the strand and that it will be added in such a way that it does not intherently affect the electrical/superconductive characteristics of the conductor. (Note that some of Sampson's data indicates that the addition of copper improves stability no matter where it is added)

The normal state resistivity and thermal conductivity of copper are about 3 onders of magninde betrer than for the NbTi superconductor. Thus adding copper will improve the ability of the conductor to remove hear and will reduce the heat generated. The effect of the superconductor is somewhat different from that of the copper, but increasing the amount of superconductor will also improve stability. First of all, more superconductor (of the same quality) means a higher crivical current and a larger temperature margin. Second, the specific heat of the superconductor is about 8 times larger than that of the copper. Thus, a conductor with more superconductor should be able to sustain a larger energy input without quenching. Finally, the addition of superconductor should make the cable more rigid mecthanically and thus aid in resisting local forces.

The clear solution to achieving greater stability under most circumstances would thus be to increase both the quantities of superconductor and of copper. Unfortunately, we are consurained to a magnet 
having a fixed conductor cross section and a fixed load line so that only a variation in the ratio is possible.

\section{MINIMUM PROPAGATING ZO'}

To estimate the size of a minimum propagating zone we use a formula similar to formulas 5.2 and 5.10 from Wilson (Ref. 4).

$$
L=\frac{2}{\mathrm{~J}_{\mathrm{c}}}\left(\frac{3 \mathrm{k}_{\mathrm{eff}}}{\rho_{\mathrm{eff}}}-\Delta \mathrm{T}\right)^{0.5}
$$

This formula assumes some current sharing in the copper and differs from the standard formulas by a scale factor. The values of MPZ will be different from those found from the formula 5.2 in Wilson by a factor of 2.45 .

To determine the variation of the MPZ with copper to superconductor racio it is necessary first to determine the critical current and the experature margin as functions of $R$ We begin by looking at the temperarure dependence of $\mathrm{J}_{\mathrm{c}}$. The SSC specification for $\mathrm{J}_{\mathrm{c}}$ at $5 \mathrm{~T}$ and $4.2 \mathrm{~K}$ is $2750 \mathrm{~A} / \mathrm{mm}^{2}$ for the superconductor in the strand. This value will be used as a reference point, but values of $\mathrm{J}_{\mathrm{c}}$ at $5 \mathrm{~T}$ and $4.2 \mathrm{~K}$ from 2250 to $3250 \mathrm{~A} / \mathrm{mm}^{2}$ will be included as to evaluate the impact of better or poorer superconductor on the MPZ. The upper critical field at $4.2 \mathrm{~K}, \mathrm{~J}=0$ is $10.5 \mathrm{~T}$, and the critical current decreases linearly to zero at a temperature defined by

$$
T=9.5-\frac{5.3 \mathrm{~B}}{10.5}
$$

Curves of $\mathrm{J}_{\mathrm{c}}(\mathrm{B}$ ) at constant $\mathrm{T}$ are given in Fig. 2 .

The relationship for $\mathrm{J}_{\mathfrak{c}}$ as a function of current and field can then be given as

$$
J_{c}(B, T)=J_{c}(5.4 .2)\left(\frac{10.5}{5.5}\right)\left(\frac{T+5.2 \frac{B}{10.5}-9.5}{5.2}\right)
$$


The calculation of the MPZ depends on the operaing conditions of the conductor. Taking the SSC inner cable as tie example, there are 23 strands that together carry $6500 \mathrm{~A}$ to produce a design central field of $6.6 \mathrm{~T}$ and a peak field of $7 \mathrm{~T}$. So the relation between current and field is such that $283 \mathrm{~A}$ in each 0.0328 " diameter strand gives $7 \mathrm{~T}$ in the magnet.

The current in the superconductor is related to the fraction of superconductor $\lambda$ and the copper to superconductor ratio $R$ :

$$
I_{s c}=\frac{I}{A_{s c}}=\frac{I}{\lambda A_{\text {tot }}}=\frac{B}{\lambda} 74.29=B(R+1) \cdot 74.29
$$

The MPZ can be expressed as a function of combined properties of all the materials.

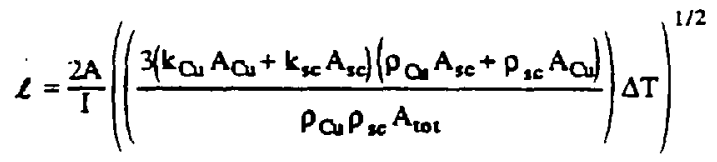

$$
\begin{aligned}
& =\frac{A_{\text {tol }}}{20.2 \mathrm{~B}}\left\{\frac{3 \mathrm{k}_{\mathrm{Cu}} A_{\mathrm{Cu}_{u}}^{2}}{\rho_{\mathrm{Cu}} A_{\mathrm{tot}}^{2}} \cdot \Delta \mathrm{T}\right\}^{1 / 2}=\frac{A_{\mathrm{tot}}}{20.2 \mathrm{~B}} \frac{R}{1+R}\left(\frac{3 k_{\mathrm{Cu}}}{\rho_{\mathrm{Cl}}} \Delta \mathrm{T}\right)^{1 / 2}
\end{aligned}
$$

In this form the MPZ depends only on the field, the temperature margin $\Delta \mathrm{T}$ and on $\mathrm{R}$.

We can combine eqs. 3 and 4 to find the maximum superconducting temperanur. $T_{m}$ and thus the temperature margin $\Delta T$

$$
\begin{gathered}
\frac{B}{\lambda} 74.29=J_{d}(5,4.2) \frac{10.5}{5.5}\left(\frac{T_{m}+5.2 B / 10.5-9.5}{5.2}\right) \\
T_{m}=9.5-B\left(0.505+\frac{74.29 \cdot 5.5 \cdot 5.2}{\lambda J_{c}(5.4 .2)-10.5}\right)
\end{gathered}
$$


The temperature margin for several conditions is shown in Fig. 3. The MPZ found from the margin to be

$$
L=\frac{(1-\lambda) 2 \cdot 0.512}{40.4 \mathrm{~B}} 3^{1 / 2}\left\{\frac{\mathrm{k}_{\mathrm{Cu}}}{\rho_{\mathrm{Cu}}}\right\}^{1 / 2}\left\{5.3-0.505 \mathrm{~B}\left(1+\frac{432.5}{\lambda_{\mathrm{J}_{\mathrm{c}}}(5,4.2)}\right)\right\}^{1 / 2}
$$

Having developed this expression for $\angle$ it is possible to explore the MPZ as various parameters change.

First, we start with values of $k_{\mathrm{Cu}}$ and $\rho_{\mathrm{Cu}}$ that are standard, and note that if we assume $\rho_{\mathrm{Cu}}$ is nor affected by field. which, as discussed above, is not a bad approximation in the range 5 to $7 \mathrm{~T}$. Then the length of the MPZ depends only on the operaing field (or equivalently the current) and $\lambda$. If we fix the operating field at various values in the operating range, say 5 to $8 \mathrm{~T}$, then it wili oe possible to explore the effect of varying $\lambda$ on the MPZ. Figures 4 and 5 are plots of MPZ as a function of copper to superconductor ratio $R$ for constant $B$ and for superconductors of different critical current density. These curves show a maximum in the length of the MPZ for copper to superconductor raios in the region berween $I$ and 2 for cerain operaing conditions. The maximum for higher fields is at lower ratios, as expected As $\mathbf{R}$ increases a point is reached where there is not sufficient superconductor to carry the current. The value of $\mathbf{R}$ at which this occurs is higher for lower fields. as shown in Fig. 6.

\section{MINLMUM ENERGY TO PRODUCE A QUENCH}

Whereas it is easy to estimate the minimum propagating zone, the minimum quench producing energy is a bit more complicated Several factors contribute to an uncertainty in MQE. The major one is the effect of helium on the transient stability. It is possible however to estimate an adiabatic MQE, i.e., in the absence of helium. 


$$
\begin{gathered}
\mathrm{MQE}=\ell \cdot A \int_{T_{-\infty}}^{T_{-}}\left(C_{C u}(1-\lambda)+C_{x} \lambda\right) \mathrm{dT} \\
=\frac{\ell \cdot A}{4}\left(\lambda C_{3 e}(4.2)+(1-\lambda) C_{C_{u}}(4.2)\right)\left(T_{m}^{4}-4.2^{4}\right)
\end{gathered}
$$

It is evident before any estimates are made that the maxima in MQE, should there be maxima, will be at lower values of $R$ than the corresponding MPZ. Plots of MQE are shown in Fig. 7. These all suggest that if this were the relevant parameter then the lowest possible ratio would be preferred.

\section{DISCUSSION}

Data presented by Sampson, et al, suggests that conductors are more stable, or at least more forgiving, if they have more copper. This snudy shows that from a theoretical approach the size of the trinimum propagating zone and the magnitude of the quench energy should be greater for lower copper io superconductor ratios. If we consider that most of the measurements have been at lower fields than those in the SSC magnets then the trend in the theoreical resuls, i.e., greater stability at lower fields for lower values of $R$ are supported somewhat, but the difference is not resolved. Since the experimental data appears to be valid and are clearly telling us something, it would seem that the area to search for an impact of $R$ on performance is in the mechanical stability characteristics of the cable. That is, in its effect on the size of the disturbance that can be created.

\section{REFERENCES}

1. Ghosh, A.K., Garber, M., Robins, K.E., and Sampson, W.B. "Training in Test Samples of Superconducting Cables for Accelerator Magnets." to be published in the Proceeding of the 1988 Applied Superconductivity Conference.

2. Private communication from M. Tigner - memos dated 19 April and 3 May 1988. 
3. King-Yuen Ng, "Results of Minimum Propagating Zone Computation," SSC-CDG Report SSC-N-521, June 1988.

4. Wilson, M.N. Superconducting.Magnets, Clarendon Press, Oxford, 1983.

\section{FIGURE CAPTIONS}

Fig. 1 The number of quenches required in test cables to reach a critical current (From Ref. 1).

Fig. 2 Critical current curves at constant temperanure as a function of field for a superconductor with $\mathrm{J}_{\mathrm{c}}(5 \mathrm{~T}, 4.2 \mathrm{k})=3000 \mathrm{~A} / \mathrm{mm}^{2}$..

Fig. 3 The temperanure margin for the superconductor under various conditions.

Fig. 4 The minimum propagaing zone for various specified crivical current densidies when the operating field is $7 \mathrm{~T}$.

Fig. 5 The minimum propagating zone for various critical current densities and various operating fields.

Fig. 6 The maximum value of copper to superconductor ratio that will allow operation at the specificed conditions.

Fig. 7 The minimura quench energy for various critical current densities when the operating field is $7 \mathrm{~T}$. 
NUMBER OF QUENCHES

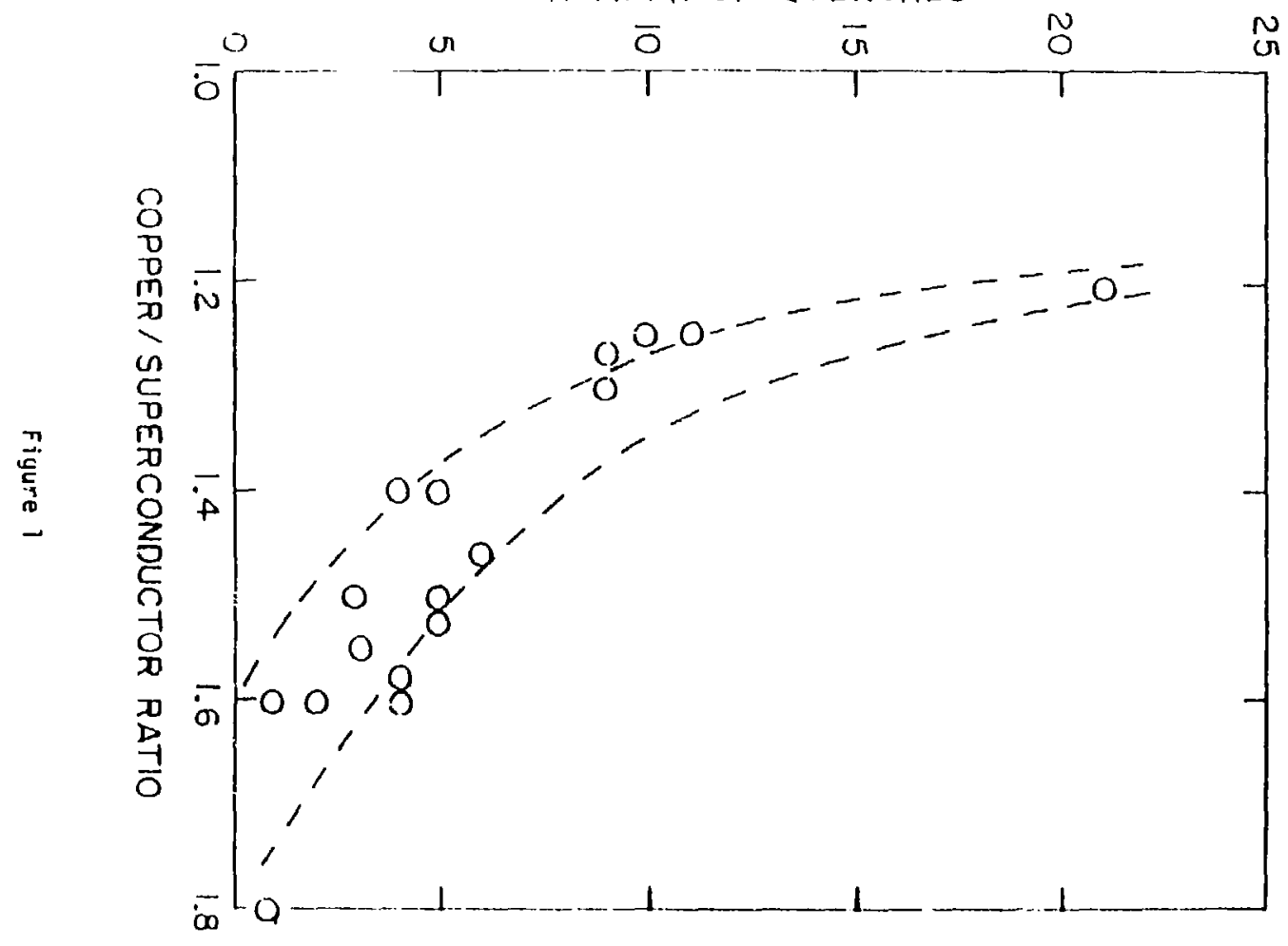




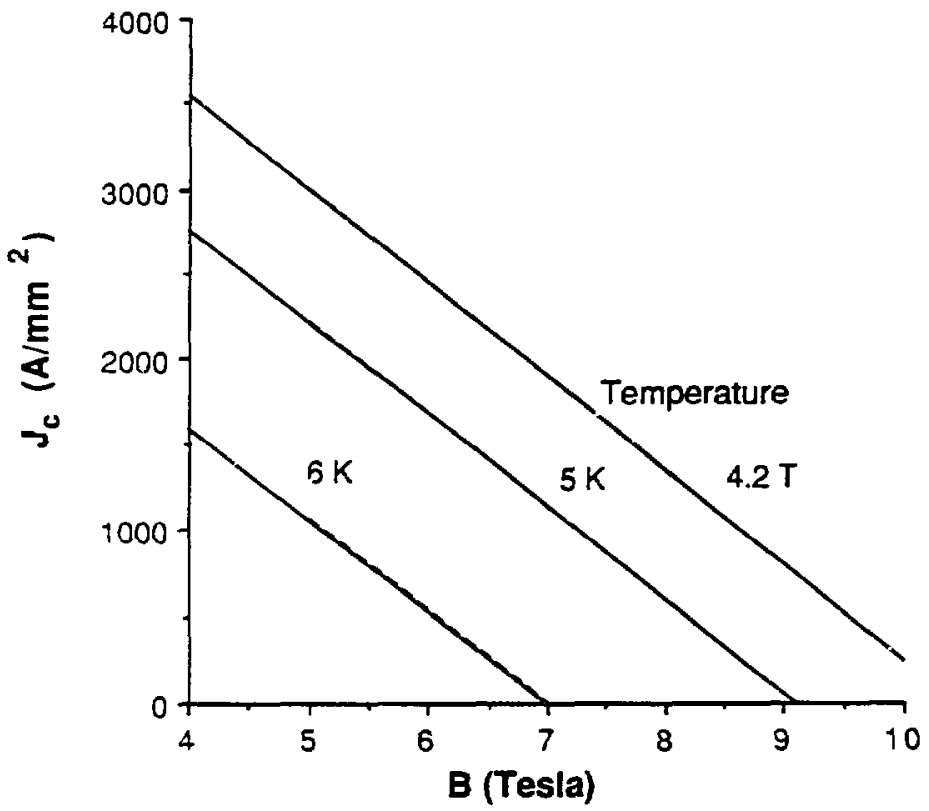

Figure 2 


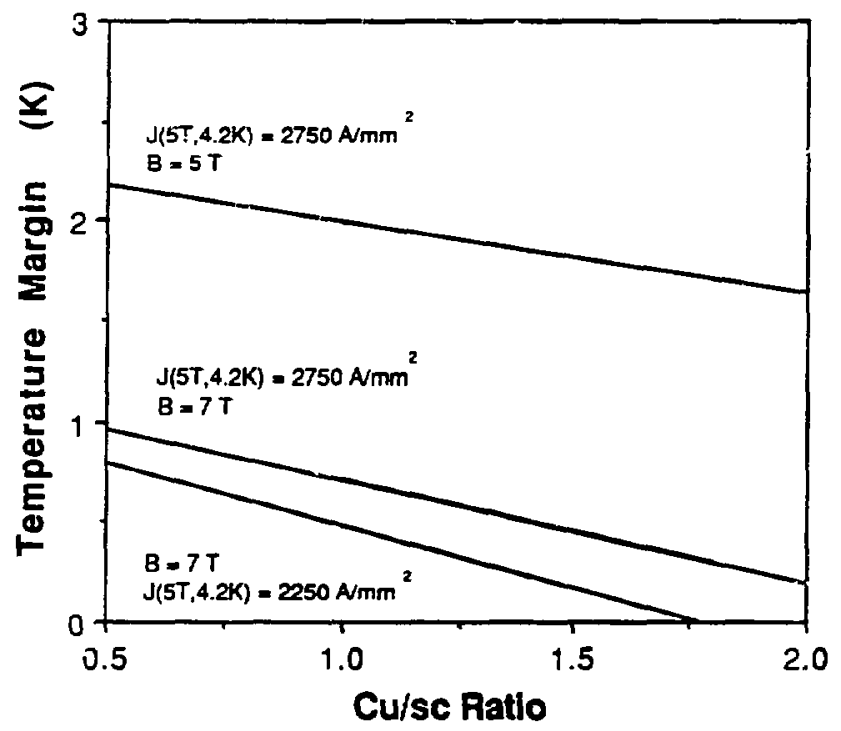

Figure 3 


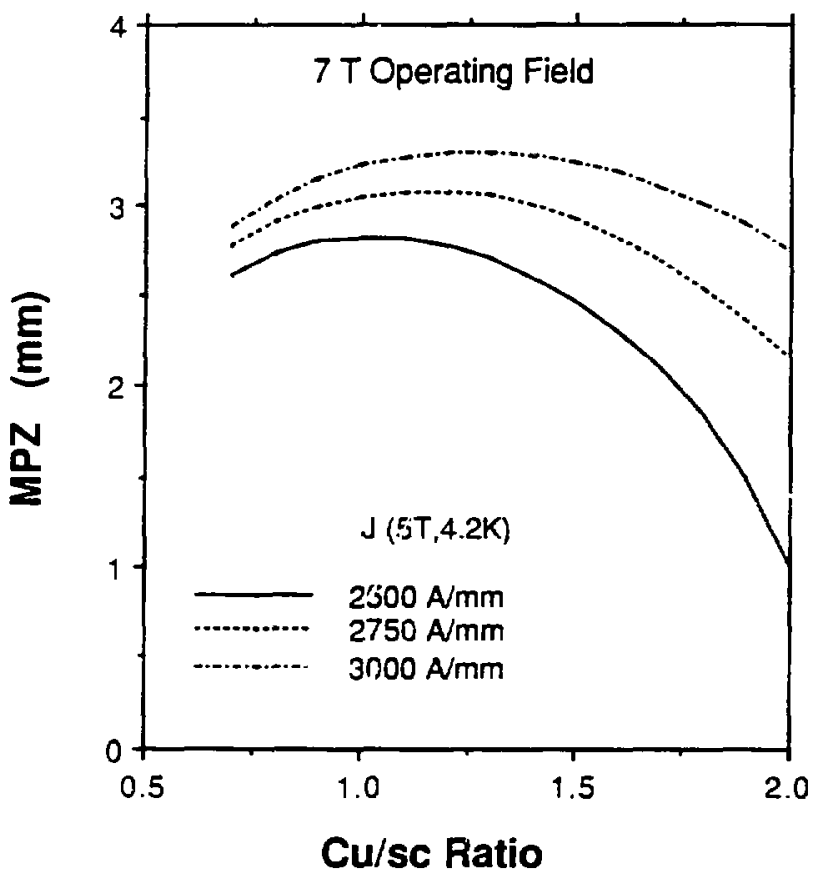

Figure 4 


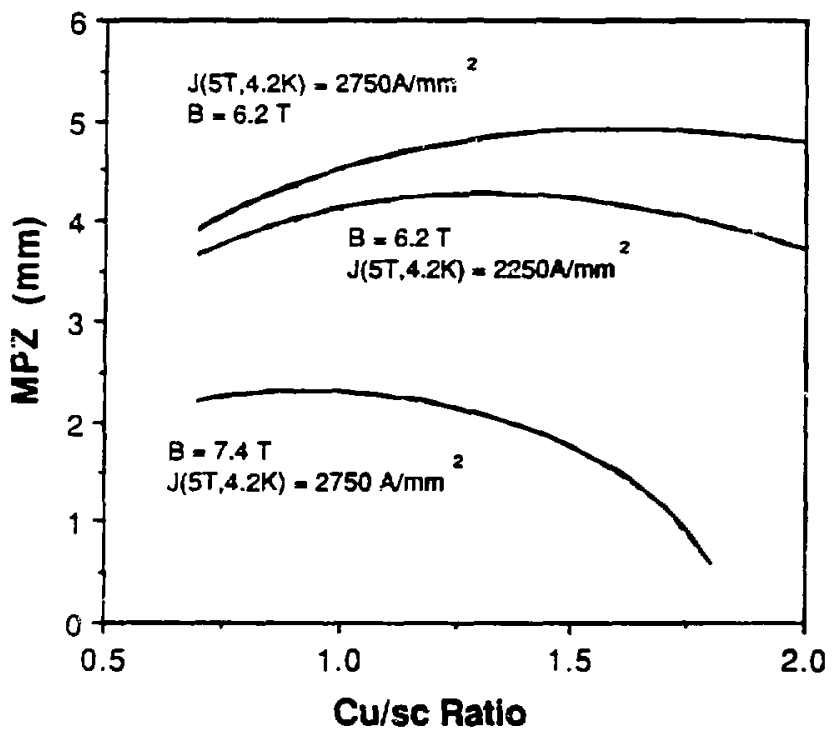

Figure 5 


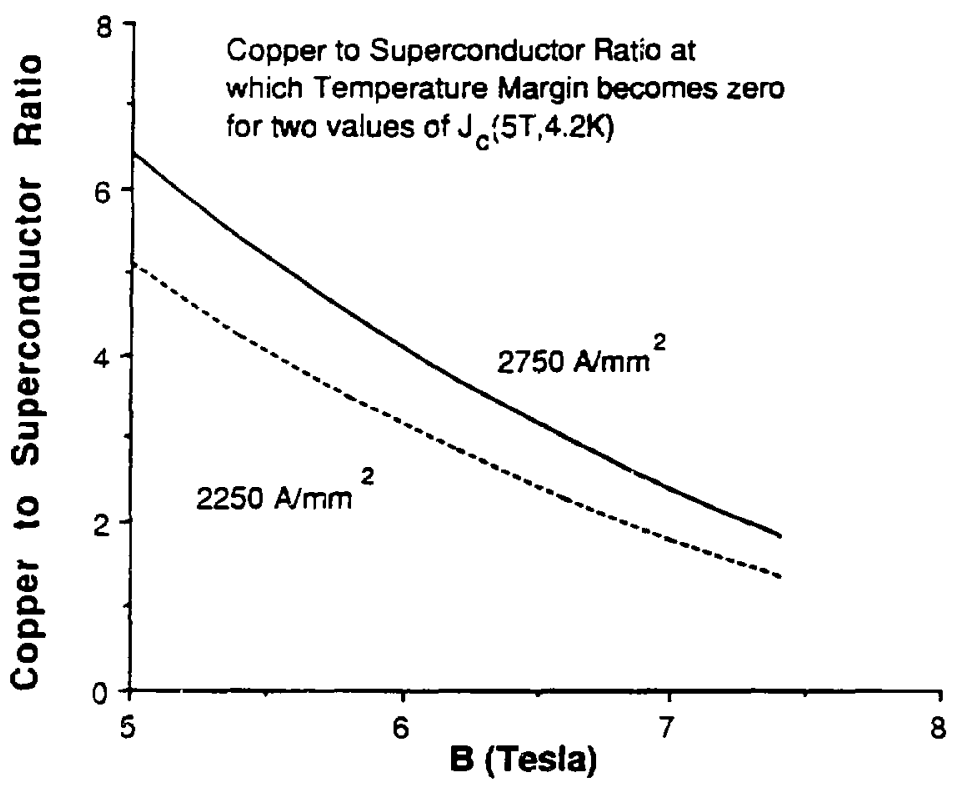

Figure 6 


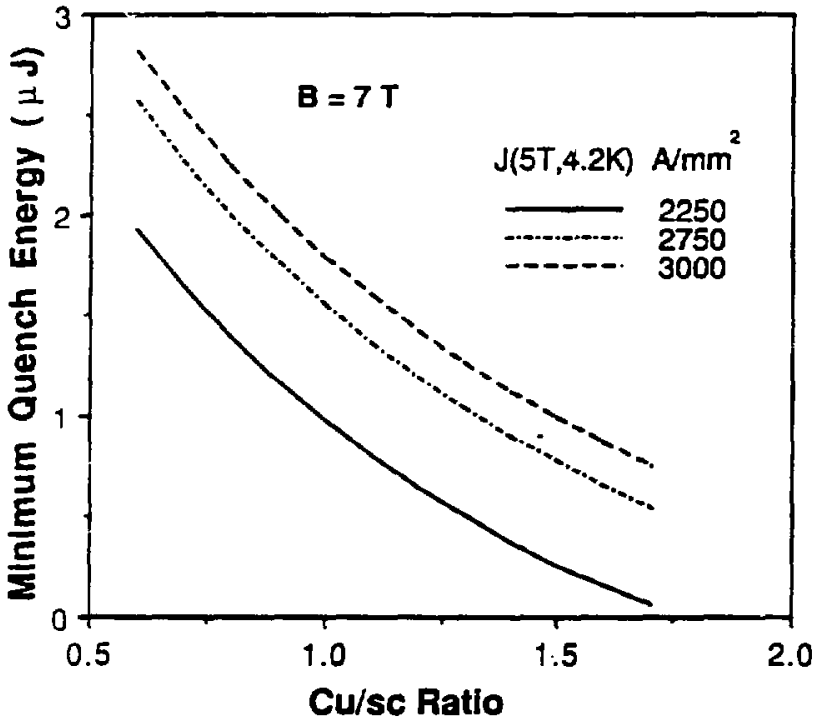

Figure 7 